\title{
Dynamics of a Levitron under a periodic magnetic forcing
}

Alberto T. Pérez and Pablo García-Sánchez

Citation: American Journal of Physics 83, 133 (2015); doi: 10.1119/1.4895800

View online: http://dx.doi.org/10.1119/1.4895800

View Table of Contents: http://aapt.scitation.org/toc/ajp/83/2

Published by the American Association of Physics Teachers

\section{Articles you may be interested in}

Spin stabilized magnetic levitation

American Journal of Physics 65, (1998); 10.1119/1.18488

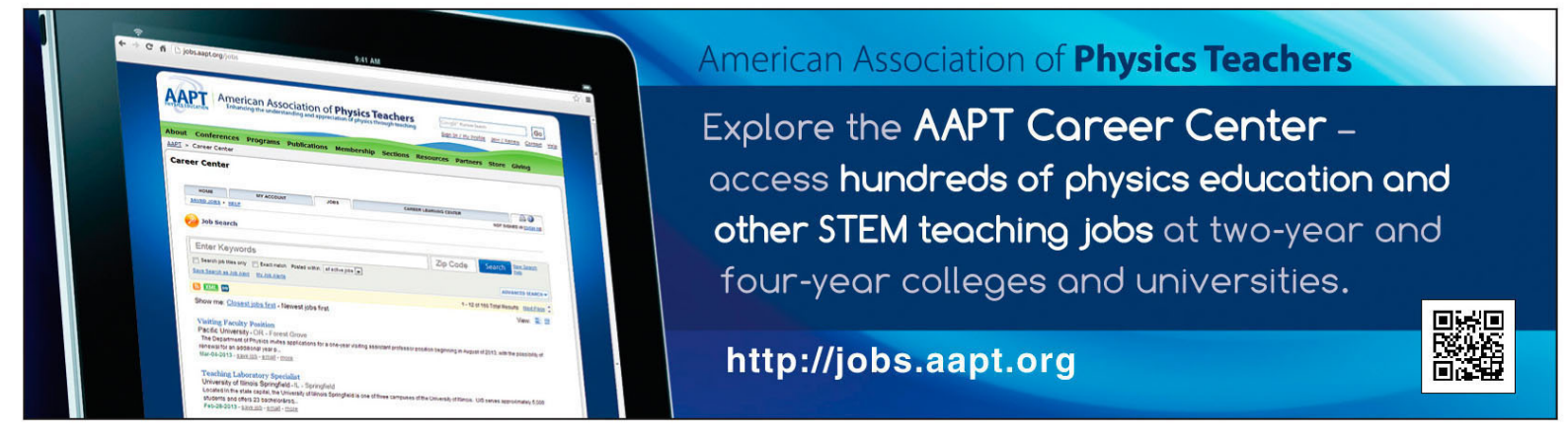




\title{
Dynamics of a Levitron under a periodic magnetic forcing
}

\author{
Alberto T. Pérez ${ }^{\mathrm{a})}$ and Pablo García-Sánchez ${ }^{\mathrm{b})}$ \\ Departamento de Electrónica y Electromagnetismo, Universidad de Sevilla, Facultad de Física, \\ Avda. Reina Mercedes s/n, 41012 Sevilla, Spain
}

(Received 17 January 2014; accepted 4 September 2014)

\begin{abstract}
The Levitron is a toy that consists of a spinning top that levitates over a magnetic base for a few minutes, until air drag decreases the spin rate below a certain limit. Stable levitation, lasting hours or even days, has been achieved for Levitrons that were externally driven by either an air jet or an alternating magnetic field. We report measurements of stable levitation for the latter case. We show that the top precession couples with the frequency of the alternating field, so that the precession period equals the period of the field. In addition, the top rotates around itself with the same period. We present numerical simulations that reproduce the essential features of this dynamics. It is also shown that the magnetic torque that drives the top is due to a misalignment between the magnetic dipole moment and the mechanical axis of the top. (c) 2015 American Association of Physics Teachers.

[http://dx.doi.org/10.1119/1.4895800]
\end{abstract}

\section{INTRODUCTION}

The Levitron is a magnetic toy that consists of a top that hovers freely above a magnetic base. The top is also made of a magnetic material and levitates due to the strong magnetic repulsion between the top and the base; the gyroscopic effect prevents the axis of the top from flipping. Stable levitation is possible only when the top is spinning within a certain range of angular velocities.

The stability of Levitron has been addressed by several authors. ${ }^{1-3}$ In order to obtain a stable levitation there are static as well as dynamic requirements. The static requirements concern the detailed functional dependence of the magnetic field; they stem from energetic considerations. But an adequate choice of the magnet design does not assure a stable levitation. The mass of the top must be judiciously adjusted if the magnetic force is to balance gravity. To this end, the toy comes with a set of washers that are used to make tiny changes to its mass.

From the dynamical point of view, there are two limits of stability for the spin rate. The levitation is not stable if the spin rate is too low, because the gyroscopic effect is not strong enough to maintain the direction of the axis of the top. On the other hand, if the top spins too fast, the axis becomes too rigid and it cannot follow the magnetic field lines. The maximum and minimum spin rates for stable levitation have been calculated using different techniques and approaches. $^{2,4-8}$

Once the top is in a stable state of levitation, air friction eventually decreases the spin rate. After about $2 \mathrm{~min}$, the angular velocity drops below the lower stability limit and the levitation ends. The company that commercializes the Levitron also sells a (patented) device called a perpetuator that compensates for the friction losses, producing a much longer levitation. ${ }^{9}$ The idea is that an alternating magnetic field induces a torque that compensates for the friction torque. To this end, the perpetuator generates an oscillating magnetic field with a horizontal component. Simon et al. ${ }^{2}$ used a similar device in their studies to counterbalance the effects of air resistance and to spin the top faster. However, whereas in the perpetuator the spin rate of the top is asynchronous with the ac applied field, Simon et al. synchronized the external field with the Levitron's spin rate by means of a feedback loop.
The aim of our paper is to analyze experimentally and numerically how an alternating horizontal magnetic field can produce a permanent levitation. We use two Helmholtz coils to produce the alternating field, and similar to the situation studied by Simon et al., the frequency of the ac magnetic field in our experiments is the same as the spin rate. Numerically, we solve the set of dynamical equations that includes the external forcing and the friction losses. We show that in order to increase the spin rate, the magnetic dipole of the top must not be exactly aligned with its symmetry axis. This deviation was already proposed by Flanders et al. as an explanation of the fact that the top precesses synchronously with the spin of the top around itself. ${ }^{7}$

This paper is organized as follows. In Secs. II and III, we present the experimental setup and results. Then, some theoretical considerations that illustrate how the external magnetic field compensates the friction losses are presented in Sec. IV. The details of the mathematical model are presented in Sec. V, while Sec. VI presents the results of the numerical simulation for different configurations and parameter sets. Finally, our conclusions are presented in Sec. VII.

\section{EXPERIMENTAL SETUP}

The experimental setup is shown in Fig. 1(a). The Levitron magnetic base is placed between two Helmholtz coils. The height of the base is chosen so that the top will spin in the central region between the coils, where the field is uniform. The magnitude and frequency of the alternating magnetic field are controlled by a function generator that supplies a sinusoidal current to the coils. A video camera records the top motion and images are transferred to a personal computer for later analysis. The precession frequency and the spin rate are determined from the videos.

The mass of the top is $13.70 \mathrm{~g}$, although the washers supplied with the Levitron can increase this mass up to $23.4 \mathrm{~g}$. The diameter of the top is $29.5 \mathrm{~mm}$ and the height of the ceramic part is $5.3 \mathrm{~mm}$. For these dimensions, the moments of inertia are $I_{1}=1.2 \times 10^{-6} \mathrm{~kg} \mathrm{~m}^{2}$ and $I_{3}=2.3 \times 10^{-6} \mathrm{~kg} \mathrm{~m}^{2}$, where $I_{3}$ is the moment of inertia about the axis of symmetry and $I_{1}$ is the moment of inertia about an axis perpendicular to the symmetry axis; these have been computed taking $21.2 \mathrm{~g}$ as the mass of the top. 
a)

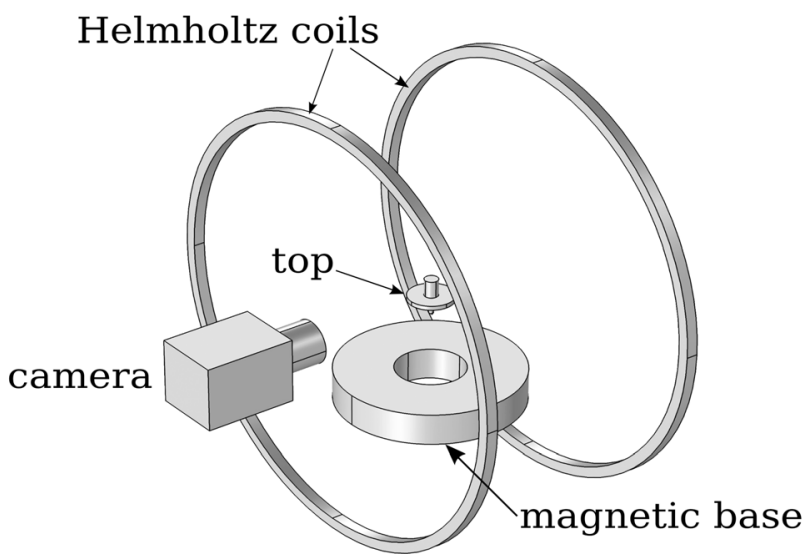

b)

\section{symmetry axis}

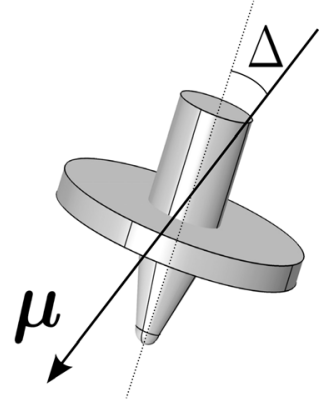

Fig. 1. (a) Schematic of the experimental setup. (b) Schematic of the top showing the deviation $\Delta$ between the symmetry axis and the axis of the magnetic dipole moment $\mu$.

We measured the magnetic field produced by the top on its axis of symmetry with the help of a tesla meter (Hall probe). Assuming that the field is that of a magnetic dipole, we obtained $\mu=0.91 \mathrm{~A} \mathrm{~m}^{2}$ for the magnetic moment of the top.

The magnetic dipole of the top is not perfectly aligned with its symmetry axis. In order to measure the angle $\Delta$ between these two directions we made use of the Helmholtz coils and a compass. We placed the top on a Petri plate, where it can move with low friction, and the plate between the Helmholtz coils. The compass was also placed between the coils, but far enough from the top so as not to interact with it. With a dc current of $3 \mathrm{~A}$ applied to the coils, they produced a magnetic field of $2.1 \mathrm{mT}$, much higher than the Earth's magnetic field. In this way, both the compass and the top aligned with the magnetic field produced by the coils. A photograph allows us to determine the angle between the magnetic orientation, given by the compass, and the symmetry axis of the top. For the top used in the experiments presented here, we find $\Delta=2.9^{\circ}=0.05 \mathrm{rad}$. Repeating this measurement for the tops of two additional Levitron sets, we obtained $1.9^{\circ}$ and $3.7^{\circ}$. Apparently, this misalignment is a random symmetry-breaking artifact of the manufacturing process. Given the variation in this angle, the efficiency of any driving system will vary from one set to another.

\section{EXPERIMENTAL RESULTS}

Before applying the external forcing to the Levitron, we took a series of recordings of the freely hovering top. The top is placed at the equilibrium height with the aid of the supplied plastic plate. There is first a transient period of stabilization in which the top spins and precesses in a complex way. When the top hovers, it rotates and precesses at different rates. At the same time, the top oscillates vertically with a well-defined frequency. After a while, precession and rotation start to synchronize and, almost at the limit of stability, when the velocity has slowed down and the angle of precession has increased, the precession and spin rates become almost the same. This coupling between precession and spinning was observed by Flanders et al. ${ }^{7}$ and was explained by considering a small angle $\Delta$ between the magnetic moment and the top axis. The synchronization of precession and rotation is clearly noticeable in our video recordings [an example (0A_90s.mov) is included in the online supplement to this article $\left.{ }^{10}\right]$.

It is important to clarify at this point that by precession we refer to the gyration of the top's symmetry axis around a vertical axis, in contrast to other definitions where precession applies only to a relatively slow gyration of the spin axis.

Figure 2 shows measurements of the spin period as a function of time in the absence of forcing. From these measurements we calculate the coefficient of friction. The typical time of levitation without forcing is around two minutes.

With the help of the Helmholtz coils, we have achieved stable levitation for times much longer than two minutes. In some cases, the levitation lasted for multiple days, although more often it lasted for a period of hours. It is not easy to obtain long stable levitations. Lifting the top in the presence of the ac magnetic field is trickier than in its absence. In addition, sometimes the hovering lasted for more than $3 \mathrm{~min}$, which is an indication of some energy transfer from the coils to the top, but the dynamics was erratic and after 5-10 min the top would fall down. We focused our study on the cases where the levitation lasted for more than $10 \mathrm{~min}$, when the features of the dynamics were well established; this was possible for ac frequencies in the range $10-50 \mathrm{~Hz}$.

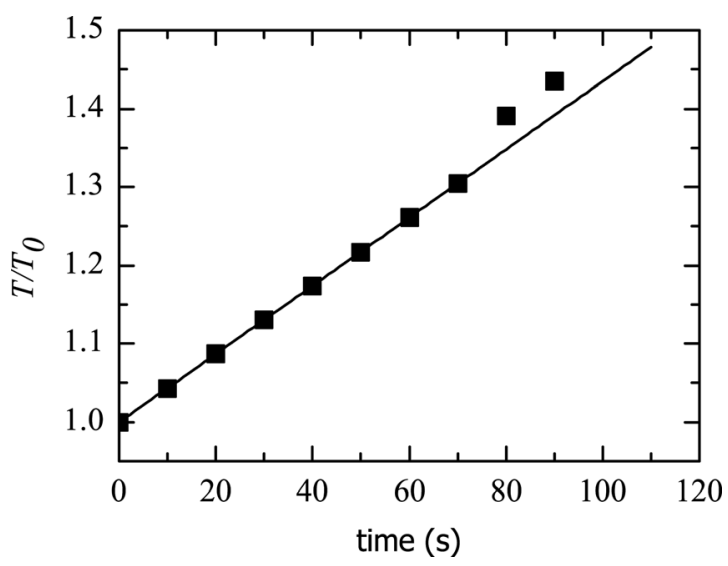

Fig. 2. Spin period as a function of time, without forcing. Values are normalized to the initial spin period $T_{0}$. 
The video recordings clearly show that the rate of precession follows the external ac field (see videos 0.40 A_30 Hz.mov and $33 \mathrm{~Hz}$ _0.51 A.mov in the online supplement). ${ }^{10}$ Figure 3 shows measurements of the top's precession frequency as a function of the frequency of the applied magnetic field (the amplitude of the applied field was $0.49 \mathrm{mT}$.) In order to achieve the steady state, the top was allowed to spin for a few minutes before capturing videos. The data are the result of averaging over at least five precession periods. It is clearly observed that the precession and the alternating field frequencies coincide within the entire range, indicating that the top precession couples to the torque exerted by the alternating field.

Along with the synchronous precession, the synchronization of the spin of the top around itself with the precession, already observed in the absence of external driving, was also observed. That is, the top rotates around itself in the same time that the axis of the top completes a turn around the vertical, and this time coincides with the period of the ac external field. Small fluctuations were observed during long recordings: sometimes the rotation of the top around itself overtakes the precession, and sometimes it lags behind. But on average both angular velocities are the same. Stable levitation was achieved with alternating fields of amplitude as small as $0.1 \mathrm{mT}$. Also, it is observed that the top height periodically oscillates with a period of around $0.78 \mathrm{~s}$.

In summary, the top motion is characterized by a precession and a rotation about its axis that are coupled to the frequency of the alternating field generated by the coils.

\section{THEORETICAL CONSIDERATIONS}

\section{A. Synchronous precession}

The synchronous precession in the absence of an external field was already observed and explained by Flanders et al. ${ }^{7}$ as due to the small misalignment between the axis of the top and its magnetic dipole moment. Figure 4(a) shows a schematic view of the relative directions of these vectors. In general, the magnetic field of the base, the top axis, and the magnetic dipole moment are not coplanar. But as shown in Fig. 4(b), there is a torque that tends to make these vectors coplanar. In the absence of friction or other losses, the result would be an oscillation of the plane containing the top axis and the dipole moment around the direction of the magnetic

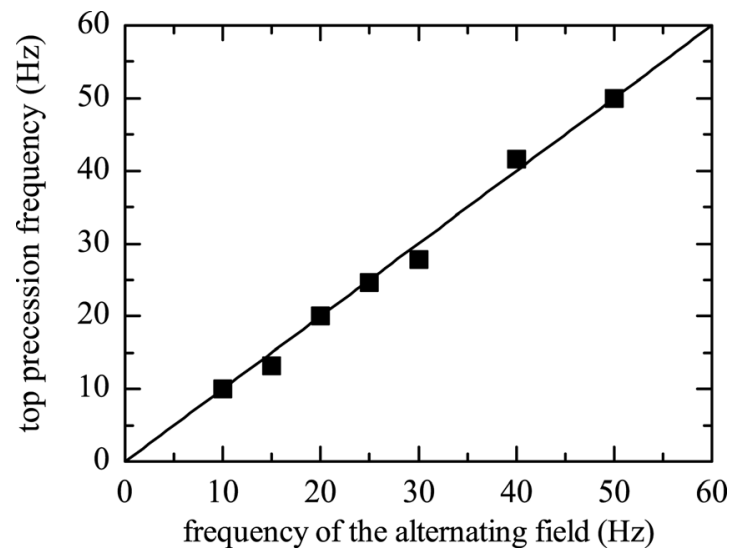

Fig. 3. Precession frequency of the top as a function of the frequency of the alternating magnetic field (magnetic field amplitude $0.49 \mathrm{mT}$ ).
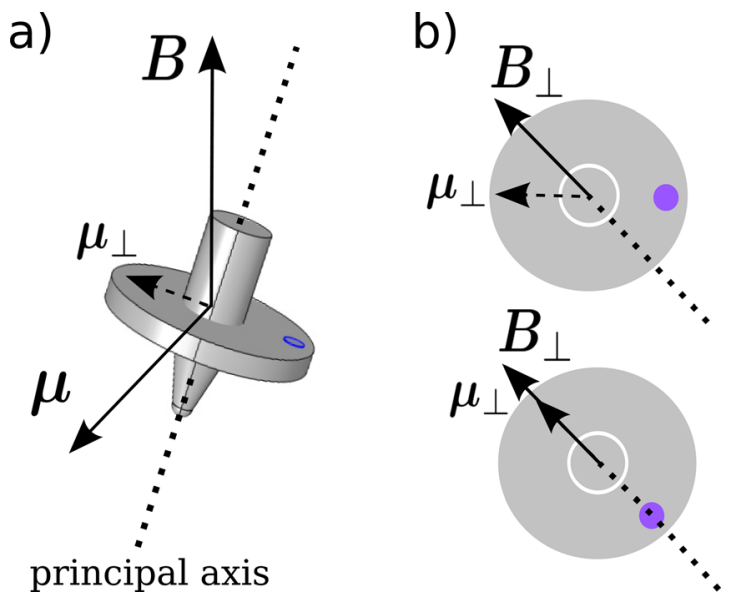

Fig. 4. (a) Relative orientation of the static magnetic field, the top axis, and the magnetic dipole moment. (b) Projection of the previous figure on a plane perpendicular to the top axis. There is a torque that tends to make $\mathbf{B}, \boldsymbol{\mu}$, and the axis of the top coplanar.

field. This oscillation would be superimposed on the precession. Actually, friction damps this mode and, after a while, the three vectors become coplanar. The result of this is that the top completes a rotation around itself in a turn of the top axis around the magnetic field, as illustrated schematically in Fig. 5.

\section{B. Coupling of precession and proper rotation with the external field}

Even if the dipole moment and the top axis were perfectly aligned $(\Delta=0)$, the precession would follow the external field. The reason for this is depicted in Fig. 6 and explained in what follows.

Suppose we align the $z$-axis with the static magnetic field due to the base, and the $y$-axis with the ac field produced by the Helmholtz coils. We write the field due to the coils as $\mathbf{B}_{H}=B_{H 0} \cos (\omega t) \mathbf{e}_{y}$, where $\omega$ is the (angular) frequency of oscillation. This field will produce a torque on the top whose $z$-component, $N_{z}$, is given by

$$
N_{z}=\mu B_{H 0} \sin \theta_{E} \cos \phi_{E} \cos (\omega t),
$$

where $\theta_{E}$ and $\phi_{E}$ are the Euler angles that define the direction of the top axis (see Fig. 6).

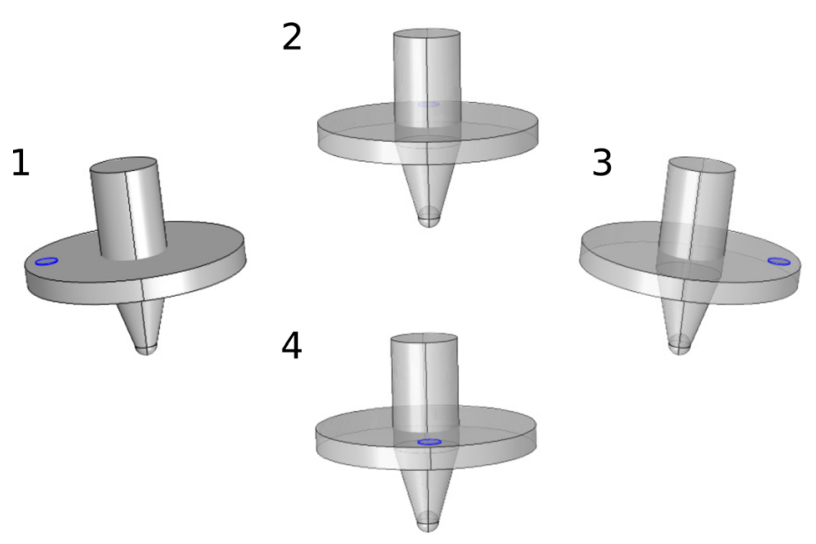

Fig. 5. The top completes a turn around its axis during a turn of the axis around the magnetic field. 


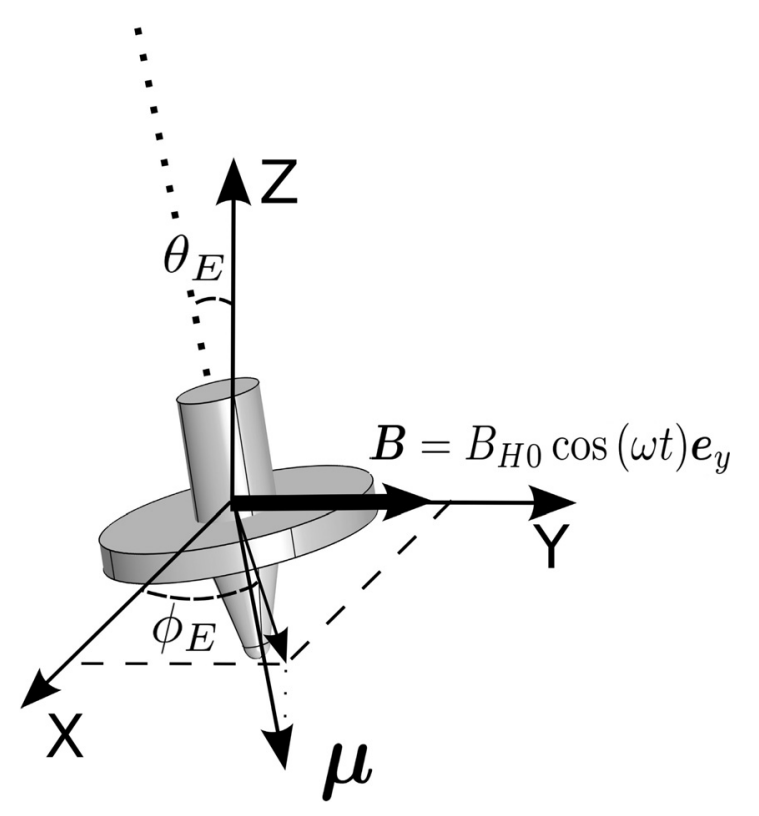

Fig. 6. Magnetic torque due to the external ac field. For simplicity, we choose $\Delta=0$.

This torque is an oscillating quantity, and its average will be different from zero only for $\phi_{E}=\omega t$, producing in that case a constant precession velocity. However, this torque would not be able to power-up the top's rotation since, for $\Delta=0$, the magnetic torque $\boldsymbol{\mu} \times \mathbf{B}$ will always be perpendicular to the axis of symmetry. On the other hand, for nonzero values of $\Delta$ there will be a nonzero component of the magnetic torque along the symmetry axis of the top that powersup the rotation and, eventually, compensates for frictional losses. Because the dipole moment, symmetry axis, and magnetic field vectors must be coplanar, the synchronization of the precession with the external field implies that the proper rotation is also synchronous with the latter.

In conclusion, the external magnetic field can counterbalance the frictional losses if the precession and the proper rotation of the top around its axis are mutually synchronized as well as synchronized with the external field.

\section{MATHEMATICAL MODEL}

\section{A. Analytical form of the magnetic field}

At the point of levitation, the magnetic field has circular symmetry up to second order in $x$ and $y,{ }^{1}$ independent of the form of the base. Following Dullin et al., ${ }^{4}$ we express the field near the levitation point as

$$
\begin{aligned}
& B_{x}=\frac{1}{2} x V^{\prime \prime}(z), \\
& B_{y}=\frac{1}{2} y V^{\prime \prime}(z), \\
& B_{z}=-V^{\prime}(z)+\frac{1}{4}\left(x^{2}+y^{2}\right) V^{\prime \prime \prime}(z),
\end{aligned}
$$

where $V$ is the magnetic scalar potential and the primes denote differentiation with respect to $z$. The potential on the $z$-axis $V_{0}(z)$ can be chosen in different ways. Again following Dullin et al., we choose the one due to a disk of radius $a$ with a hole of radius $b$ :

$$
V(z)=2 B_{0} \pi z\left(\frac{1}{\sqrt{b^{2}+z^{2}}}-\frac{1}{\sqrt{a^{2}+z^{2}}}\right) .
$$

We measured the magnetic field created by the base magnet on its axis with the help of a standard tesla meter. A nonlinear fitting allows us to determine the three parameters $a$, $b$, and $B_{0}$ that best reproduce the field. Figure 7 shows a plot of the measured magnetic field along with the field computed from Eqs. (4) and (5) using the best-fit parameters $a=5.4 \mathrm{~cm}, b / a=0.35$, and $B_{0}=7.6 \mathrm{mT}$. The quality of the fit shows that Eq. (5) satisfactorily reproduces the field on the $z$-axis. We also measured the $z$-component of the magnetic field as a function of the coordinates $x$ and $y$ near the point of levitation and found that it satisfactorily follows the quadratic law given in Eq. (4).

Assuming that $B_{z}>0$, the conditions of stability imply ${ }^{1-3}$

$$
\begin{aligned}
& V^{\prime \prime}\left(z_{c}\right)>0 \\
& V^{\prime \prime \prime}\left(z_{c}\right)<0 \\
& V^{\prime \prime \prime}\left(z_{c}\right)-\frac{1}{2} \frac{\left[V^{\prime \prime}\left(z_{c}\right)\right]^{2}}{V^{\prime}\left(z_{c}\right)}>0,
\end{aligned}
$$

where $z_{c}$ is the coordinate of the point of levitation. The first condition is required for the force between the base and the top to be repulsive. For $b / a=0.35$, the last two conditions imply

$$
\begin{aligned}
& z_{c} / a>1.057, \\
& z_{c} / a<1.134 .
\end{aligned}
$$

The trapping region-where these conditions are met and stable levitation is achieved-is rather small (less than half a centimeter); this is one of the features that makes it difficult to master the toy.

\section{B. Dynamical equations}

The dynamics of the top is described by six coordinates: the Cartesian coordinates $(x, y$, and $z$ ) of its center of mass, and three angles that give the spatial orientation. The classical definition of Euler angles leads to a system of equations that is singular when the top is spinning around the axis of

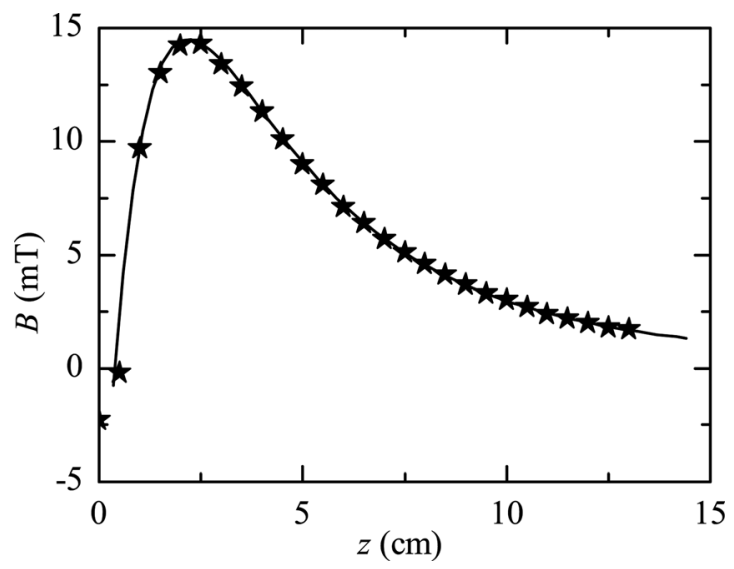

Fig. 7. Graph of $B$ on the $z$-axis as a function of height above the Levitron base. Symbols correspond to the measured field and the continuous line to the fit to Eq. (4). 
symmetry of the field. In order to avoid this singularity, we use a version of the yaw-pitch-roll sequence, in which the top orientation is given by three angles $(\psi, \theta$, and $\phi)$ defined as follows. The roll angle $\phi$ represents a clockwise rotation about the $x$-axis; the pitch angle $\theta$ is a counterclockwise rotation about the $y$-axis; and the yaw angle $\psi$ is a clockwise rotation about the $z$-axis. The coordinates of any point of the top with respect to a fixed frame of reference can be found by applying the matrix of rotation $R(\psi, \theta, \phi)$ to the coordinates of the point in a frame comoving with the top. The detailed expression for this matrix can be found in the Appendix.

The angular velocity in the top frame can be expressed as a function of the three angles just defined. For the component along the axis of symmetry we have $\Omega_{3}=-\dot{\psi}-\sin \theta \dot{\phi}$. For the axes perpendicular to it, we have $\Omega_{1}=-\cos \theta \cos \psi \dot{\phi}-$ $\sin \psi \dot{\theta}$ and $\Omega_{2}=-\cos \theta \sin \psi \dot{\phi}+\cos \psi \dot{\theta}$.

Due to the complexity of the geometrical configuration, the Lagrangian method is the fastest and safest way of obtaining the dynamical equations. The Lagrangian is given by

$$
\begin{aligned}
\mathcal{L}(x, y, z, \psi, \theta, \phi) & \\
= & \frac{1}{2} m\left(\dot{x}^{2}+\dot{y}^{2}+\dot{z}^{2}\right)+\frac{1}{2} I_{1}\left(\dot{\theta}^{2}+\cos ^{2} \theta \dot{\phi}^{2}\right) \\
& +\frac{1}{2} I_{3}(\dot{\psi}+\sin \theta \dot{\phi})^{2}-U(x, y, z, \psi, \theta, \phi),
\end{aligned}
$$

where $m$ is the top mass and $U$ is the potential energy function.

The Helmholtz coils couple to the residual transverse magnetization of the top. In order to describe the effect of this transverse magnetization, we assume that the axis of magnetization makes an angle $\Delta$ with respect to the mechanical axis of symmetry, as in Fig. 1(b). Taking the $x z$-plane as the plane that contains the magnetic dipole in the frame comoving with the top, the magnetic dipole moment can be written as

$$
\boldsymbol{\mu}=-\left(\mathbf{n}_{z} \cos \Delta+\mathbf{n}_{x} \sin \Delta\right) \mu,
$$

where $\mu$ is the magnitude of the dipole. The minus sign is to indicate that the dipole moment is pointing downwards, a condition required for equilibrium if we assume that the base magnetic field is pointing upwards. The unit vectors $\mathbf{n}_{x}$ and $\mathbf{n}_{z}$ are given by (see the Appendix)

$$
\begin{aligned}
\mathbf{n}_{x}= & (\cos \psi \cos \theta) \mathbf{e}_{x}-(\cos \phi \sin \psi+\cos \psi \sin \phi \sin \theta) \mathbf{e}_{y} \\
& +(\sin \phi \sin \psi-\cos \phi \cos \psi \sin \theta) \mathbf{e}_{z}, \\
\mathbf{n}_{z}= & (\sin \theta) \mathbf{e}_{x}+(\cos \theta \sin \phi) \mathbf{e}_{y}+(\cos \theta \cos \phi) \mathbf{e}_{z},
\end{aligned}
$$

where $\mathbf{e}_{x}, \mathbf{e}_{y}$, and $\mathbf{e}_{z}$ are the Cartesian unit vectors in the laboratory frame.

To complete the expression for the Lagrangian, we write the potential energy of the system as follows:

$$
\begin{aligned}
U= & m g z+\mu \cos \Delta\left(B_{x} \sin \theta+B_{y} \cos \theta \sin \phi+B_{z} \cos \theta \cos \phi\right) \\
& +\mu \sin \Delta\left[B_{x} \cos \psi \cos \theta-B_{y}(\cos \phi \sin \psi+\cos \psi \sin \phi \sin \theta)\right. \\
& \left.+B_{z}(\sin \phi \sin \psi-\cos \phi \cos \psi \sin \theta)\right] .
\end{aligned}
$$

Since we use MATLAB to solve the set of differential equations, it is more convenient to express the equations as first-order differential equations, which MATLAB's solvers can handle directly. To this end, we will switch from the Lagrangian to the Hamiltonian formalism.

The momenta conjugate to the angular coordinates are

$$
\begin{aligned}
& p_{\psi}=\frac{\partial \mathcal{L}}{\partial \dot{\psi}}=I_{3}(\dot{\psi}+\sin \theta \dot{\phi}) \\
& p_{\theta}=\frac{\partial \mathcal{L}}{\partial \dot{\theta}}=I_{1} \dot{\theta} \\
& p_{\phi}=\frac{\partial \mathcal{L}}{\partial \dot{\phi}}=I_{3} \sin \theta \dot{\psi}+\left(I_{3} \sin ^{2} \theta+I_{1} \cos ^{2} \theta\right) \dot{\phi}
\end{aligned}
$$

giving the Hamiltonian ${ }^{4}$

$$
\begin{aligned}
\mathcal{H}\left(x, y, z, p_{x}, p_{y}, p_{z}, \psi, \theta, \phi\right) & =\frac{1}{2 m}\left(p_{x}^{2}+p_{y}^{2}+p_{z}^{2}\right)+\frac{1}{2 I_{1}}\left[p_{\theta}^{2}+\frac{\left(p_{\phi}-p_{\psi} \sin \theta\right)^{2}}{\cos ^{2} \theta}\right] \\
& +\frac{p_{\psi}^{2}}{2 I_{3}}+U(x, y, z, \psi, \theta, \phi) .
\end{aligned}
$$

To perform numerical integrations it is convenient to define dimensionless variables, whose values will typically be of the order of unity. We take $\tau=\sqrt{I_{1} /\left(\mu B_{0}\right)}$ as the time scale, and the parameter $a$ defined in Eq. (5) as the distance scale. Hamilton's equations then become

$$
\begin{aligned}
& \dot{x}=p_{x}, \quad \dot{y}=p_{y}, \quad \dot{z}=p_{z} \\
& \dot{p}_{x}=-\frac{\partial U}{\partial x}, \quad \dot{p}_{y}=-\frac{\partial U}{\partial y}, \quad \dot{p}_{z}=-\frac{\partial U}{\partial z}, \\
& \dot{\psi}=\lambda p_{\psi}+\frac{\sin \theta}{\cos ^{2} \theta}\left(p_{\psi} \sin \theta-p_{\phi}\right) \\
& \dot{\theta}=p_{\theta}, \\
& \dot{\phi}=-\frac{1}{\cos ^{2} \theta}\left(p_{\psi} \sin \theta-p_{\phi}\right) \\
& \dot{p}_{\psi}=-\frac{\partial U}{\partial \psi}, \\
& \dot{p}_{\theta}=\frac{1}{\cos ^{3} \theta}\left(p_{\psi} \sin \theta-p_{\phi}\right)\left(p_{\phi} \sin \theta-p_{\psi}\right)-\frac{\partial U}{\partial \theta} \\
& \dot{p}_{\phi}=-\frac{\partial U}{\partial \phi},
\end{aligned}
$$

where the potential energy $U$ is

$$
\begin{aligned}
U= & G z+\frac{1}{\Lambda} \cos \Delta\left(B_{x} \sin \theta+B_{y} \cos \theta \sin \phi+B_{z} \cos \theta \cos \phi\right) \\
& +\mu \sin \Delta\left[B_{x} \cos \psi \cos \theta\right. \\
& -B_{y}(\cos \phi \sin \psi+\cos \psi \sin \phi \sin \theta) \\
& \left.+B_{z}(\sin \phi \sin \psi-\cos \phi \cos \psi \sin \theta)\right]
\end{aligned}
$$

and the dimensionless magnetic field is given by Eqs. (2)-(4) with $V(z)$ the dimensionless form (with $b$ and $z$ both scaled by $a$ )

$$
V(z)=2 \pi z\left(\frac{1}{\sqrt{b^{2}+z^{2}}}-\frac{1}{\sqrt{1+z^{2}}}\right) .
$$


In these equations, there are three dimensionless parameters defined by

$$
\begin{aligned}
& \Lambda=\frac{m a^{2}}{I_{1}}, \quad G=\frac{g I_{1}}{a \mu B_{0}}, \\
& \lambda=\frac{I_{1}}{I_{3}} .
\end{aligned}
$$

A full description of the top dynamics under magnetic forcing must include a time-dependent magnetic field and a friction mechanism. Because the field produced by the Helmholtz coils is uniform in the region of levitation, we can write this additional magnetic field as

$$
\delta \mathbf{B}=\epsilon B_{0} \cos (\omega t) \mathbf{e}_{y},
$$

where we have taken the $y$-axis to be the axis of the Helmholtz coils.

When the top is spinning and levitating, the only source of friction comes from the air, and we assume that this friction is proportional to the angular velocity about the symmetry axis of the top. Under this assumption, Eq. (25) must be replaced by

$$
\dot{p}_{\psi}=-\frac{\partial U}{\partial \psi}-\nu p_{\psi},
$$

where $\nu$ is a dimensionless friction coefficient.

\section{NUMERICAL RESULTS}

\section{A. Dimensionless parameters}

At the point of equilibrium, Eq. (28), for $\Delta=0$, requires

$$
\frac{m g a}{\mu B_{0}}=\Lambda G=V^{\prime \prime}\left(z_{c}\right) .
$$

Since conditions (9) and (10) require $1.057<z_{c}<1.134$, the values of $V^{\prime \prime}(z)$ at the ends of the interval of stability give the limits of mass for a stable levitation. For $z_{c}=1.096, V^{\prime \prime}\left(z_{c}\right)=1.6185$, which gives $m=21.2 \mathrm{~g}$.

The characteristics of the top described in Sec. II, along with the value of $B_{0}$, give the following dimensionless parameters for stable levitation: $\Lambda=31.3$ and $G=0.0315$. We also find that the ratio of the moments of inertia is $\lambda=0.52$, while the time scale is $\tau=13.2 \mathrm{~ms}$.

The friction coefficient can be obtained from the data in Fig. 4. The angular velocity of the top closely follows the exponential law $\dot{\psi}=\dot{\psi}_{0} e^{-\nu_{f} t}$ with a coefficient $\nu_{f}=4.35$ $\times 10^{-3} \mathrm{~s}^{-1}$. The corresponding dimensionless parameter is $\nu=\nu_{f} \tau=5.74 \times 10^{-5}$.

\section{B. Results without forcing; magnetic moment aligned to the axis of the top}

The system of Eqs. (20)-(27) was solved using MAтLAв. ${ }^{11}$ In particular, we used the function ODE45, the standard MATLAB solver for systems of ordinary differential equations. In order to test our script we ran a series of tests without friction $(\nu=0)$, without misalignment $(\Delta=0)$, and without forcing $(\epsilon=0)$.

Figure 8 is a plot of the trajectory of the top in the $x z$-plane for $\dot{\psi}_{0}=2$ and 160 dimensionless time units. The excursions

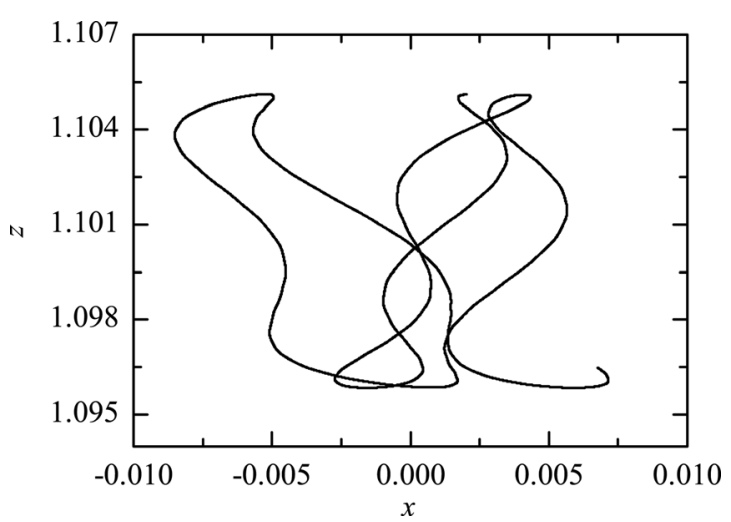

Fig. 8. Trajectory of the top in the $x z$-plane. The dimensionless parameters are $\lambda=0.52, b=0.35, \Lambda=51.3, G=0.0315, \Delta=0, \nu=0$, and $\epsilon=0$. The initial angular velocity is $\dot{\psi}_{0}=2.0$.

of the top in the $x$-direction are determined by the initial conditions.

The top can levitate within a certain range of spinning velocities. Below and above the limiting values, stable levitation is not possible and the top falls down. The limits of stable levitation have been computed by several authors using different approaches. ${ }^{2,4-8}$ All of them give the same value for the minimum spin rate for levitation:

$$
\omega_{\min }=2 \sqrt{\frac{I_{1} \mu B}{I_{3}^{2}}},
$$

which, in terms of our dimensionless quantities, becomes

$$
\omega_{\min }=2 \lambda \sqrt{\left|V^{\prime}\left(z_{c}\right)\right|} .
$$

The exact value of the upper limit depends on the analytical form of the magnetic field and on the approximations made in its computation. The different results can be written as

$$
\omega_{\max }=\gamma \frac{(B \mu)^{3 / 2}}{I_{3} g m^{1 / 2}},
$$

which, in terms of our dimensionless quantities, becomes

$$
\omega_{\max }=\gamma \sqrt{\left|V^{\prime}\left(z_{c}\right)\right|^{3}} \frac{\lambda}{G \sqrt{\Lambda}},
$$

where $\gamma$ is a factor of order unit (for example, $\gamma=4 / \sqrt{27} \approx$ 0.77 for Dullin et al., ${ }^{4}$ and $\gamma=0.86$ for Genta et al. ${ }^{5}$ )

In order to determine the lower limit of the stability range, we introduced a nonzero friction coefficient in the numerical computation. The top angular velocity slows down until, at a certain velocity, the top "flies apart" (i.e., the top position changes beyond the stability limits). For $\lambda=0.52, b=0.35$, $\Lambda=51.3, G=0.0315$, and $\Delta=0$, we obtain $\omega_{\min }=1.2$ for the minimum velocity for levitation, a value to be compared with 1.24, as predicted from Eq. (36).

The upper limit can be obtained by introducing a negative friction coefficient. This has the effect of slowly accelerating the top until it reaches an unstable angular velocity. For the same set of parameters we find $\omega_{\max }=3.5$, which is higher than prediction of Eq. (38), of around 2.4. A careful numerical study of the instability, which is not the main purpose of our work, would be required to compute a more accurate upper limit. 
Simon et al. ${ }^{2}$ made two testable predictions for the motion of the top without friction. The first is that the angular precession frequency is

$$
\omega_{p}=\frac{\mu B}{I \omega},
$$

where $\omega$ is the angular frequency of rotation around the axis of the top. In terms of dimensionless variables, this relation becomes

$$
\omega_{p}=\frac{\lambda}{\omega} .
$$

We have verified this prediction for several values of the top spin velocity. In fact, the precession is not uniform, and the angular velocity of precession oscillates with an amplitude that depends on the initial conditions. The above relation holds if we average both angular velocities in time. For an example, see the video animation psi2eps0theta05.mov in the supplementary material. ${ }^{10}$

We note that this behavior is numerically encountered only when the angle $\Delta$ is set to zero, and that we have not observed this behavior in our experiments, a consequence, we believe, of the misalignment between the axis of the top and its magnetic dipole moment. This misalignment is to some degree unavoidable.

The second prediction by Simon et al., which is also made by other authors, 5,6 is that the top oscillates in $z$ with a frequency

$$
\Omega_{z}=\sqrt{\frac{1}{m} \mu \frac{\partial^{2} B_{z}}{\partial z^{2}}},
$$

which corresponds to

$$
\Omega_{z}=\sqrt{\frac{V^{\prime \prime \prime}\left(z_{c}\right)}{\Lambda}},
$$

in our dimensionless variables. This is due to the fact that, close to the levitating point, the equation of motion in the $z$-direction is that of a harmonic oscillator.

Figure 9 is a plot of the $z$-coordinate of the top as a function of time for the same conditions as in the previous plots. The motion is that of a harmonic oscillator with a dimensionless period of 61.3. This value compares satisfactorily with

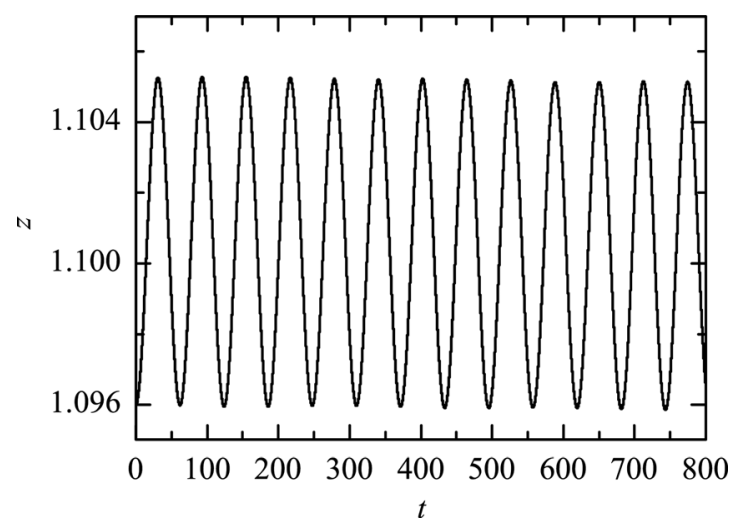

Fig. 9. Vertical coordinate $z$ of the top as a function of time. The parameters are $\lambda=0.52, b=0.35, \Lambda=51.3, G=0.0315, \Delta=0, \nu=0$, and $\epsilon=0$. The initial angular velocity is $\dot{\psi}_{0}=2.0$. The period of oscillation is 61.3 . the value 62 predicted by Eq. (42). (As mentioned in Sec. III, we observed in experiments that the top height oscillates with a period of around $0.78 \mathrm{~ms}$, which corresponds to a dimensionless period of 59.1).

\section{Magnetic moment out of the axis of symmetry; synchronous precession}

As we have already mentioned, Flanders et $a{ }^{7}{ }^{7}$ showed that a small deviation of the magnetic moment from the symmetry axis of the top synchronizes the precession and the rotation of the top, in a way similar to the motion of the moon around the earth. In order to test this prediction, we ran several simulations for different values of $\Delta$, the angle between the magnetic moment and the top axis. For $\Delta \sim 0.01$ a partial synchronization is observed. For $\Delta \geq 0.05$ the synchronization is clearly observed. Because the exact motion is not steady the synchronization is not perfect, but it takes place on average (see the video psi 2eps0delta05fland 02 . mov in the supplementary material). ${ }^{10}$

Interestingly, Flanders et al. discussed the stability limits for nonzero $\Delta$ and found that, for the case $I_{1}<I_{3}$, the minimum velocity for stable levitation is higher than in the case of $\Delta=0$. We have checked the stability limits for $\Delta=0.05$ and obtained a maximum spinning velocity of $\dot{\psi}_{\max }=3.46$ and a minimum of $\dot{\psi}_{\min }=1.74$, a higher value than the result in the previous section for $\Delta=0$.

For $\Delta=0$, the origin of the upper stability limit is linked to Eq. (40). When the top spins fast, the precession is too slow to keep the top oriented to the magnetic field direction. ${ }^{2}$ It is not clear whether this mechanism is present when $\Delta \neq 0$, because in this case the faster the spinning the faster the precession. The persistence of an upper limit for stable levitation calls for further analysis of the stability limits, which is beyond the scope of this paper.

The degree of synchronization depends on the initial conditions. Flanders et al. ${ }^{7}$ deduced that synchronization implies that

$$
(1-\lambda) \omega^{2} \sin \theta_{E} \cos \theta_{E}=\lambda \sin \left(\theta_{E}+\Delta\right),
$$

where the condition is expressed in terms of our dimensionless parameters and $\theta_{E}$ is the polar angle in the Euler convention. This expression is deduced under the assumption of a uniform magnetic field.

In order to achieve exact synchronization in a given simulation, the initial conditions must be compatible with Eq. (43). Figure 10 shows the angular velocity of precession as a function of time for a set of initial conditions that fulfills Eq. (43). In this case $\theta_{0}=0.02, \phi_{0}=0.0, \dot{\phi}_{0}=0.05, \dot{\theta}_{0}=0.0$, and $\dot{\psi}_{0}=-2.1511$. The synchronization is clearly observed, although perfect synchronization has not been achieved. In any case, the main effect of the misalignment between the magnetic and the mechanical axis is clearly demonstrated.

If we introduce a nonzero friction coefficient, the oscillations observed in Fig. 10 tend to disappear, in agreement with the experimental observations.

\section{Motion under ac magnetic driving}

Once we checked that our numerical code satisfactorily reproduces the main theoretical predictions in the absence of external driving, we proceeded to simulate the effect of an applied ac field produced by the Helmholtz coils. 


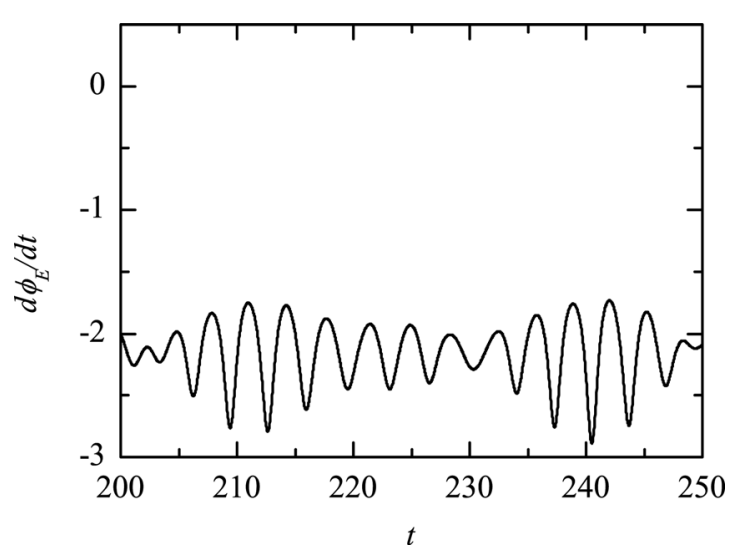

Fig. 10. Angular velocity of precession as a function of time. The parameters are $\lambda=0.52, b=0.35, \Lambda=51.3, G=0.0315, \Delta=0.05, \nu=0$, and $\epsilon=0$. The initial angular velocity is $\dot{\psi}_{0}=-2.1511$.

Since the magnetic torque is $\boldsymbol{\mu} \times \mathbf{B}$, the torque will be perpendicular to the top axis if the magnetic dipole and the mechanical axis of symmetry are exactly aligned. In that case, an external ac field would not sustain the levitation. The small misalignment characterized by the angle $\Delta$ makes it possible to have a nonzero component of the magnetic torque along the axis of rotation of the top. This is reflected mathematically in the appearance of a dependence on $\psi$ in the magnetic energy [Eq. (28)]. Note that this dependence disappears for $\Delta=0$. Roughly speaking, the component of the magnetic torque on the top axis is $\mu_{x} B_{y}$. Since $B_{y}$ is a sinusoidal function of time, $\mu_{x}$ must oscillate at the same frequency in order to have a nonzero average. As a consequence, the precession of the top is locked to the frequency of the ac magnetic field.

Figure 11 shows the angular velocity $\Omega_{3}$ as a function of time for a value of $\epsilon=0.052$, which corresponds to an amplitude of $0.40 \mathrm{mT}$ (a positive friction coefficient $\nu=5.74 \times 10^{-5}$ is also included). The angular velocity oscillates around 2.0 , but it maintains its average value, thus reflecting a balance between the driving magnetic torque and the friction torque. The amplitude of the oscillations decreases slowly in time and tends to a steady value. Figure 12 displays the trajectory of the top in the $x z$-plane. The overall behavior is similar to that in Fig. 8, but there is a small oscillation in the trajectory that reflects the frequency

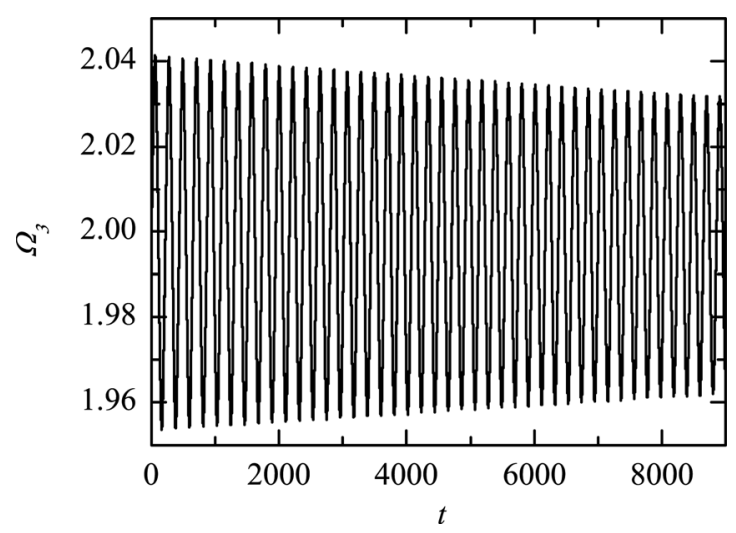

Fig. 11. Angular velocity of the top as a function of time when an ac transverse magnetic field is applied. The parameters are $\lambda=0.52, b=0.35$, $\Lambda=51.3, G=0.0315, \Delta=0.05, \nu=5.74 \times 10^{-5}$, and $\epsilon=0.052$. The initial angular velocity is $\dot{\psi}_{0}=2.0$.

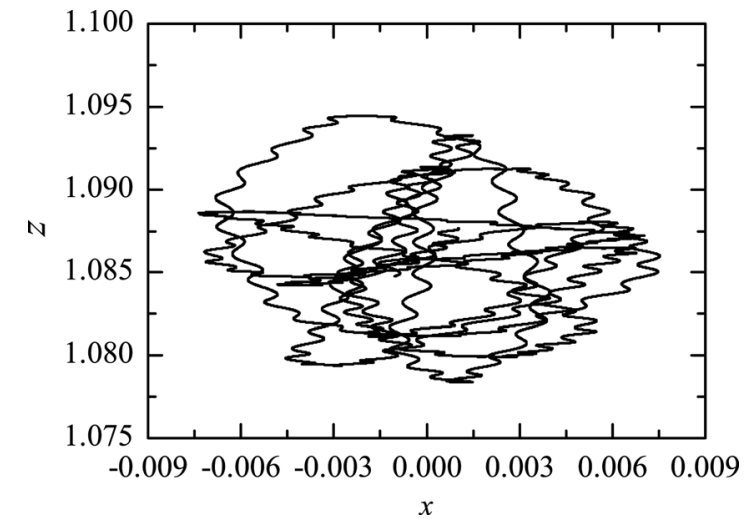

Fig. 12. Trajectory of the top in the $x z$-plane. The parameters are $\lambda=0.52$, $b=0.35, \Lambda=51.3, G=0.0315, \Delta=0.05, \nu=5.74 \times 10^{-5}$, and $\epsilon=0.052$. The initial angular velocity is $\dot{\psi}_{0}=2.0$.

of the driving magnetic field. Video psi2eps052x3.mov in the supplementary material ${ }^{10}$ is an animation of the motion for these parameters.

The driving torque can compensate for the friction losses only if there is an average synchronization between the ac magnetic field and the top precession. This synchronization is illustrated in Fig. 13, which plots the precession velocity as a function of time. The average value is exactly 2.0, the top angular velocity. We have observed that the initial angular velocity of the top must be very close to the angular frequency of the magnetic field in order to obtain a stable levitation. For example, for a magnetic field angular frequency $\omega=2.0$ and an initial top spinning velocity $\dot{\psi}=2.04$ we obtained, after a transient, a sustained motion with $\left\langle\dot{\phi}_{E}\right\rangle=2.0$. However, a value of $\dot{\psi}=2.05$ produces an unstable motion and the top falls after a while. This is consistent with our experimental experience: it is rather difficult and takes several tries to set the top in stable levitation when the ac field is applied.

The synchronization is better illustrated in Fig. 14, where the supplementary angle to the angle of precession is plotted along with the phase of the alternating magnetic field. If the difference between these two angles is zero the torque takes its maximum value. Although sometimes the precession lags behind the field phase and at other instants it overtakes the field phase, the two quantities are in phase on average. In all the simulations conducted with $\Delta \neq 0$ and a nonzero ac field,

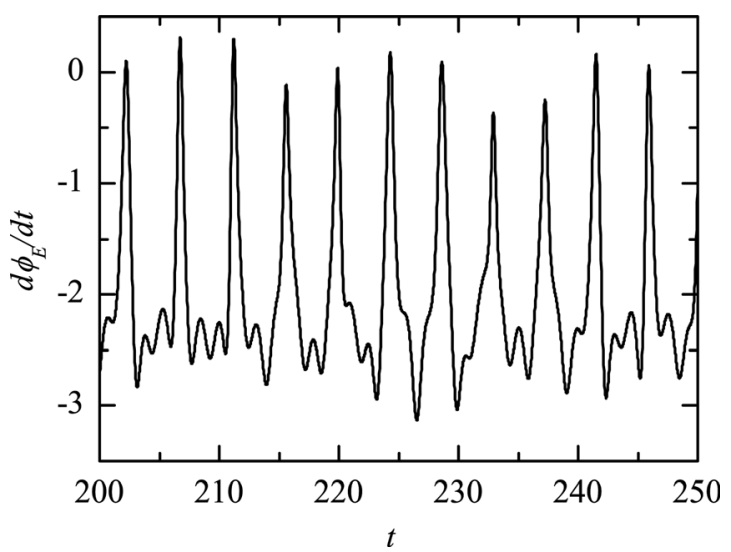

Fig. 13. Angular velocity of precession as a function of time. The parameters are $\lambda=0.52, b=0.35, \Lambda=51.3, G=0.0315, \Delta=0.05, \nu=5.74 \times 10^{-5}$, and $\epsilon=0.052$. The initial angular velocity is $\dot{\psi}_{0}=2.0$. 


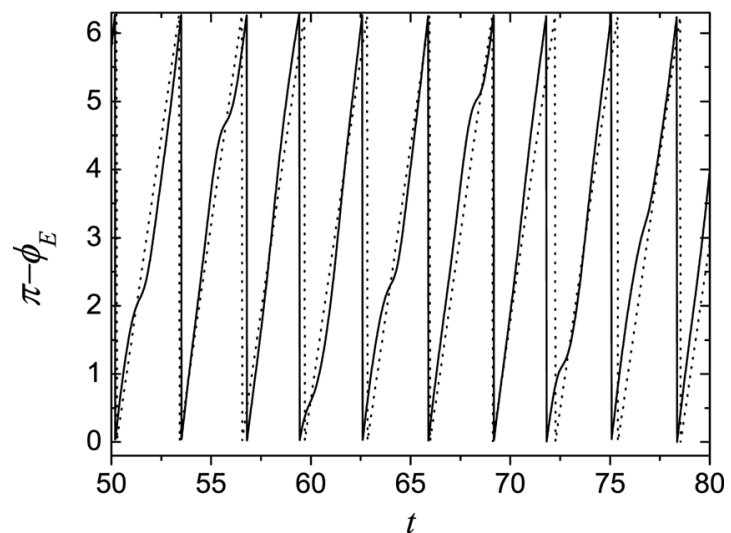

Fig. 14. Supplementary angle to the angle of precession (solid line) and phase of the external magnetic field (dotted line) as a function of time. When the angle and the phase are the same, the magnetic torque is at a maximum. The parameters are $\lambda=0.52, b=0.35, \Lambda=51.3, \quad G=0.0315$, $\Delta=0.05, \nu=5.74 \times 10^{-5}$, and $\epsilon=0.052$. The initial angular velocity is $\dot{\psi}_{0}=2.0$.

the angular velocity of rotation of the top about its own axis is, on average, the same as the angular velocity of precession.

As found experimentally (Fig. 3), the precession frequency remains equal to the magnetic field angular frequency for a wide range of frequencies, in the range of stable spinning velocities of the top. Consistently, this precession frequency does not change with the amplitude of the ac magnetic field. Since an increase in $\epsilon$, the amplitude of the ac magnetic field, would induce a stronger torque, the top adjusts its angle of inclination $\theta_{E}$ in order to reduce the torque. Our simulations show that $\theta_{E}$ increases slowly when the magnetic field increases. For $\omega=2$, and in the range of $0.026<\epsilon<0.065$, where stable levitation was obtained, we found the following fit:

$$
\left\langle\theta_{E}\right\rangle=0.15 \epsilon+0.034 \text {. }
$$

For values of $\epsilon<0.026$, the average torque created by the ac magnetic field cannot compensate friction. For $\epsilon>0.065$ the ac magnetic field strongly perturbs the top motion and the top falls down.

\section{CONCLUSION}

Experiments with a Levitron subjected to a horizontal alternating magnetic field show that stable levitation can be achieved with amplitudes of the alternating field as small as $0.1 \mathrm{mT}$. The top precession synchronizes with the proper rotation and couples to the frequency of the alternating field. The resulting magnetic torque balances the air drag on the top. We present the equations for the top dynamics including the air drag and the ac magnetic forcing. Numerical solutions of the equations for the top dynamics are in accordance with experimental observations and show that a misalignment between the magnetic and the mechanical axis of symmetry is needed in order to achieve permanent levitation.

\section{ACKNOWLEDGMENTS}

This work has been carried out with financial support from the Spanish Ministerio de Ciencia y Tecnología (MCYT) under Research Project No. FIS2011-25161, and Junta de Andalucía under Research Project Nos. P10-FQM-5735 and P09-FQM-4584.

\section{APPENDIX: ROTATION MATRICES}

Consider a point $\mathbf{x}$ with coordinates $(x, y, z)$ described as a column vector. The transformation from one coordinate system to another can be represented by a matrix equation of the form

$$
\mathbf{x}^{\prime}=R(\psi, \theta, \phi) \mathbf{x}
$$

If we identify the fixed system with $\mathbf{x}$ and the top system with $\mathbf{x}^{\prime}$, the matrix $R$ completely describes the relative orientation of the two systems. The rotation matrix $R$ contains three independent angles. There are many possible choices for these angles. We choose the yaw-pitch-roll angles since this set does not lead to singular equations when the top is spinning vertically.

In this convention, the matrix $R(\psi, \theta, \phi)$ is built in the following way:

$$
R(\psi, \theta, \phi)=R_{z}(\psi) R_{y}(\theta) R_{x}(\phi),
$$

where the matrices $R_{x}, R_{y}$, and $R_{z}$ are rotations about the $x, y$, and $z$ axes, respectively, and are given by

$$
\begin{aligned}
& R_{x}=\left(\begin{array}{ccc}
1 & 0 & 0 \\
0 & \cos \phi & -\sin \phi \\
0 & \sin \phi & \cos \phi
\end{array}\right), \\
& R_{y}=\left(\begin{array}{ccc}
\cos \theta & 0 & -\sin \theta \\
0 & 1 & 0 \\
\sin \theta & 0 & \cos \theta
\end{array}\right), \\
& R_{z}=\left(\begin{array}{ccc}
\cos \psi & -\sin \psi & 0 \\
\sin \psi & \cos \psi & 0 \\
0 & 0 & 1
\end{array}\right) .
\end{aligned}
$$

The explicit expression of $R(\psi, \theta, \phi)$ is

$$
R=\left(\begin{array}{ccc}
\cos \psi \cos \theta & -\cos \phi \sin \psi-\cos \psi \sin \phi \sin \theta & \sin \phi \sin \psi-\cos \phi \cos \psi \sin \theta \\
\cos \theta \sin \psi & \cos \phi \cos \psi-\sin \phi \sin \psi \sin \theta & -\cos \psi \sin \phi-\cos \phi \sin \psi \sin \theta \\
\sin \theta & \cos \theta \sin \phi & \cos \phi \cos \theta
\end{array}\right) .
$$

The inverse matrix $R^{-1}=R^{T}$ transforms coordinates in the top system into coordinates in the fixed system: $\mathbf{x}=R^{-1}(\psi, \theta, \phi) \mathbf{x}^{\prime}$. The unit vector in the direction of the axis of the top in the top system of coordinates is $\mathbf{x}^{\prime}=(001)^{T}$. Therefore, the vector $\mathbf{n}_{z}$ is given by the third column of $R^{-1}$, which is the third row of $R$; that is, 


$$
\mathbf{n}_{z}=(\sin \theta) \mathbf{e}_{x}+(\cos \theta \sin \phi) \mathbf{e}_{y}+(\cos \theta \cos \phi) \mathbf{e}_{z} .
$$

Similarly, $\mathbf{n}_{x}$ is given by the first row of $R$ :

$$
\begin{aligned}
\mathbf{n}_{x}= & (\cos \psi \cos \theta) \mathbf{e}_{x}-(\cos \phi \sin \psi+\cos \psi \sin \phi \sin \theta) \mathbf{e}_{y} \\
& +(\sin \phi \sin \psi-\cos \phi \cos \psi \sin \theta) \mathbf{e}_{z} .
\end{aligned}
$$

The supplementary material ${ }^{10}$ includes three animations of the simulated top motion. The top model is built using MATLAB functions developed by Land. ${ }^{12}$ This model is then rotated using the transformation $\mathbf{x}(\mathbf{t})=R^{-1}(\psi(t)$, $\theta(t), \phi(t)) \mathbf{x}^{\prime}$, where the angles $\psi(t), \theta(t)$, and $\phi(t)$ are obtained from the numerical solution of the system of differential equations.

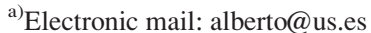

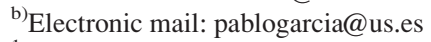

${ }^{1}$ M. V. Berry "The Levitron (TM): an adiabatic trap for spins," Proc. R. Soc. London, Ser. A 452, 1207-1220 (1996).
}

${ }^{2}$ Martin D. Simon, Lee O. Helfinger, and S. L. Ridgway, "Spin stabilized magnetic levitation,” Am. J. Phys. 65(4), 286-292 (1997).

${ }^{3}$ T. B. Jones, Masao Washizu, and Roger Gans, "Simple theory for the Levitron,” J. Appl. Phys. 82, 883-888 (1997).

${ }^{4}$ Holger R. Dullin and Robert W. Easton, "Stability of Levitrons," Physica D 126, 1-17 (1999).

${ }^{5}$ G. Genta, C. Delprete, and D. Rondano, "Gyroscopic stabilization of passive magnetic Levitation," Meccanica 34, 411-424 (1999).

${ }^{6} \mathrm{~S}$. Gov, S. Shtrikman, and H. Thomas, "On the dynamical stability of the hovering magnetic top," Physica D 126, 214-224 (1999).

${ }^{7}$ P. Flanders, S. Gov, S. Shtrikman, and H. Thomas, "On the spinning motion of the hovering magnetic top," Physica D 126, 225-235 (1999).

${ }^{8}$ A. San Miguel, "Numerical integration for the dynamics of the heavy magnetic top," Phys. Lett. A 335, 235-244 (2005).

${ }^{9}$ E. W. Hones and W. G. Hones, "Electromagnetic drive method and apparatus for driving a rotationally stabilized magnetic levitated object," U.S. patent No. 5,883,454 (1999).

${ }^{10}$ See supplementary material at http://dx.doi.org/10.1119/1.4895800 for six videos, three showing experimental observations and three showing numerical simulations.

${ }^{11}$ The MATLAB programs are available upon request to author A.T.P. at alberto@us.es.

${ }^{12}$ Bruce R. Land, "A hierarchical graphics modeler using Matlab," $<$ http:// www.nbb.cornell.edu/neurobio/land/projects/hierarchy/>.

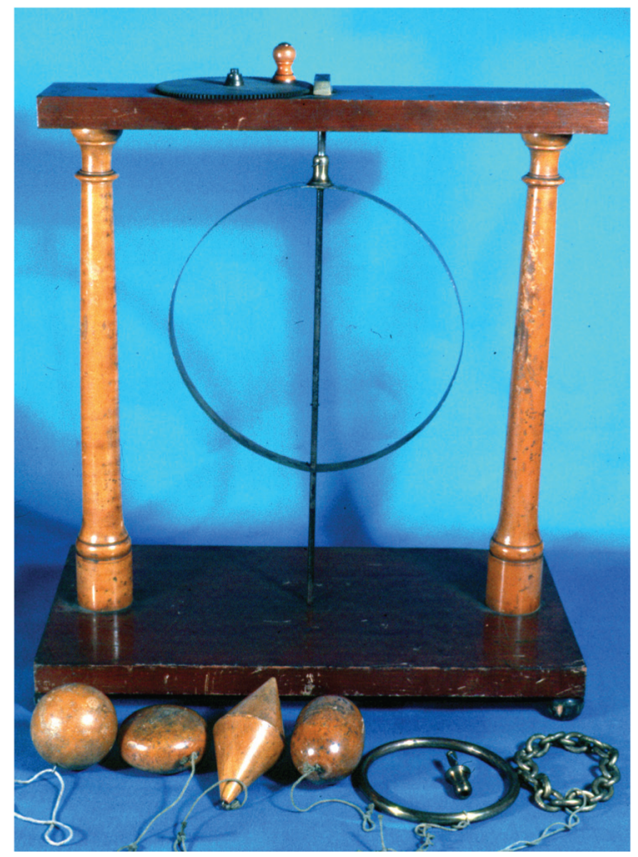

\section{Rotator}

When I photographed this rotator and its accompanying accessories in 1978, it was in the collection of Wittenberg University. It is marked "Chamberlain" but by 1860 the firm was owned by E.S. Ritchie of Boston. The Ritchie catalogue of that year has the following entry: "Whirling Machine; illustrating central and centrifugal forces, the flattening of the poles of the planets, the revolution of bodies on their shortest diameter; mahogany frame, 20 inches long by 18 high, brass geared wheels and movable spindle; with eight illustrations, viz. a double elastic brass ring, glass globes for mercury, \&., oblate and prolate spheres, cylinder, brass disk, brass ring, ring of chain, ... \$8.00" (Notes and photograph by Thomas B. Greenslade, Jr., Kenyon College) 